\title{
139 Determination of myocardial OEF with a CMR method in a stenotic dog study
}

\author{
Kyle S McCommis*, Robert J Gropler, Jie Zheng \\ From $11^{\text {th }}$ Annual SCMR Scientific Sessions \\ Los Angeles, CA, USA. 1-3 February 2008
}

\section{Introduction}

Recently, we have shown that a CMR method can noninvasively determine the oxygen extraction fraction (OEF) in dogs during hyperemia [1,2] with assumed myocardial blood volume (MBV) values. The purpose of this study is to determine the effect of quantified MBV to the calculation of myocardial OEF during pharmacologically induced hyperemia in coronary artery stenotic dogs.

\section{Methods}

Thirteen dogs were divided into four groups (Table 1). Stenosis was created by using an occluder in the proximal left anterior descending coronary artery (LAD) in an open-chest model. Single-slice T2-weighted images were gathered by use of a 2-D segmented turbo spin-echo (TSE) sequence with double inversion recovery pulses. T2 maps can be generated with these T2-weighted images with different TE's acquired in a single breathhold. This T2-imaging was performed several times during rest and during either Dipyridamole or Dobutamineinduced hyperemia. Using a two-compartment model validated in normal dogs [1], the OEF during hyperemia can be determined assuming resting OEF of 0.6, which is based on OEF values measured in normal dogs using an arterial and coronary sinus blood sampling approach at rest [3]. It is assumed that this value changes little with moderate stenosis [4], but for severe stenoses, this may be a potential source of error. The model also requires input of MBV values both at rest and during pharmaceutical hyperemia. These values were determined with a validated first-pass perfusion cardiovascular magnetic resonance (CMR) method [4]. OEF values were determined in the stenotic LAD region and a remote normal left-circumflex (LCX) subtended region.

Mallinckrodt Institute of Radiology, Washington University School of Medicine, St. Louis, MO, USA

\begin{tabular}{lll}
\multicolumn{2}{l}{ Table $\mathbf{1}$ Table of dog groups } \\
\hline Group $(\boldsymbol{n})$ & Stenosis & Hyperemia \\
\hline $1(4)$ & $70 \%$ & Dipyridamole \\
$2(3)$ & $90 \%$ & Dipyridamole \\
$3(3)$ & $50 \%$ & Dobutamine \\
$4(3)$ & $70-90 \%$ & Dobutamine \\
\hline
\end{tabular}

\section{Results}

There is no significant change in OEF values calculated using assumed MBV and MBV measured with the quantitative CMR first-pass method. Nevertheless, the former slightly overestimated the later $(0.3 \%$ in LAD-perfused and $5.8 \%$ in LCX-perfused regions) (Figure 1). This difference may explain why the former method slightly overestimated the "true" OEF values measured in normal dogs using the arterial-venous blood sampling approach [1]. The changes in OEF with measured MBV are shown in Figure 2. With the injection of Dipyridamole, while normal LAD region demonstrates reduced OEF of 0.31 (48\% reduction), OEF in the LAD region changes to 0.54 ( $11 \%$ decrease) and 0.6 ( $0 \%$ change) in dogs with $70 \%$ and $90 \%$ stenosis, respectively. During the Dobutamine-induced hyperemia, dogs with stenosis had slight changes in OEF in the LAD region, but an increase to 0.69 ( $14 \%$ increase) and decrease to 0.46 (23\%) in the LCX region with $50 \%$ and $70-90 \%$ stenosis, respectively $(P=\mathrm{NS})$. This is consistent with our previous observation [2].

\section{Conclusion}

Using the two-compartment model and CMR methods, the effect of MBV quantification is subtle in the calculation of myocardial OEF. In dogs with varying severities of coronary artery stenosis, regional differences in OEF during the hyperemia can be readily detected using this CMR method. 




Figure 1 Bland-Altman plots of the difference in OEF calculated with assumed MBV and measured MBV.



Figure 2 Off changes during hyperemia The impact of myocardial blood volume determination to a CMR method was evaluated in the estimation of myocardial OEF during hyperemia in a canine stenosis model. The effect of blood volume is subtle and regional myocardial OEF can be detected.

Published: 22 October 2008

\section{References}

1. Zheng J, et al: Magn Reson Med 2004, 51:718-26.

2. McCommis KS, et al: Magn Reson Imaging 2007.

3. Zhang H, et al: J Magn Reson Imaging 2007, 26:72-9.

4. McCommis KS, et al: J Cardiovasc Magn Reson 2007, 9:785-92.

5. Buck A, et al: J Nucl Med 1991, 32:1950-7.
doi:10.1186/1532-429X-10-S1-A40

Cite this article as: McCommis et al: 139 Determination of myocardial

OEF with a CMR method in a stenotic dog study. Journal of

Cardiovascular Magnetic Resonance 2008 10(Suppl 1):A40.
Submit your next manuscript to BioMed Central and take full advantage of:

- Convenient online submission

- Thorough peer review

- No space constraints or color figure charges

- Immediate publication on acceptance

- Inclusion in PubMed, CAS, Scopus and Google Scholar

- Research which is freely available for redistribution

Submit your manuscript at www.biomedcentral.com/submit 